\title{
Late normal tissue response in the rat spinal cord after carbon ion irradiation
}

Maria Saager ${ }^{1,2,3^{*}}$, Peter Peschke, ${ }^{3,4}$, Thomas Welzel ${ }^{1,3}$, Lifi Huang ${ }^{2,3}$, Stephan Brons ${ }^{3,5}$, Rebecca Grün ${ }^{6}$, Michael Scholz ${ }^{6}$, Jürgen Debus ${ }^{1,3}$ and Christian P. Karger ${ }^{2,3}$

\begin{abstract}
Background: The present work summarizes the research activities on radiation-induced late effects in the rat spinal cord carried out within the "clinical research group ion beam therapy" funded by the German Research Foundation (DFG, KFO 214).

Methods and materials: Dose-response curves for the endpoint radiation-induced myelopathy were determined at 6 different positions (LET 16-99 keV/ $\mu \mathrm{m}$ ) within a $6 \mathrm{~cm}$ spread-out Bragg peak using either 1, 2 or 6 fractions of carbon ions. Based on the tolerance dose $\mathrm{TD}_{50}$ of carbon ions and photons, the relative biological effectiveness (RBE) was determined and compared with predictions of the local effect model (LEM I and IV). Within a longitudinal magnetic resonance imaging (MRI)-based study the temporal development of radiation-induced changes in the spinal cord was characterized. To test the protective potential of the ACE (angiotensin converting enzyme)-inhibitor ramipril ${ }^{\mathrm{TM}}$, an additional dose-response experiment was performed.

Results: The RBE-values increased with LET and the increase was found to be larger for smaller fractional doses. Benchmarking the RBE-values as predicted by LEM I and LEM IV with the measured data revealed that LEM IV is more accurate in the high-LET, while LEM I is more accurate in the low-LET region. Characterization of the temporal development of radiation-induced changes with MRI demonstrated a shorter latency time for carbon ions, reflected on the histological level by an increased vessel perforation after carbon ion as compared to photon irradiations. For the ACE-inhibitor ramipril ${ }^{\mathrm{TM}}$, a mitigative rather than protective effect was found.

Conclusions: This comprehensive study established a large and consistent RBE data base for late effects in the rat spinal cord after carbon ion irradiation which will be further extended in ongoing studies. Using MRI, an extensive characterization of the temporal development of radiation-induced alterations was obtained. The reduced latency time for carbon ions is expected to originate from a dynamic interaction of various complex pathological processes. A dominant observation after carbon ion irradiation was an increase in vessel perforation preferentially in the white matter. To enable a targeted pharmacological intervention more details of the molecular pathways, responsible for the development of radiation-induced myelopathy are required.
\end{abstract}

Keywords: Carbon ion therapy, Relative biological effectiveness (RBE), Rat spinal cord, Magnetic resonance imaging, Radiation induced myelopathy

\footnotetext{
* Correspondence: m.saager@dkfz.de

${ }^{1}$ Department of Radiation Oncology, University Hospital of Heidelberg, Heidelberg, Germany

2Department of Medical Physics in Radiation Oncology (E040), German

Cancer Research Center (DKFZ), Im Neuenheimer Feld 280, 69120

Heidelberg, Germany

Full list of author information is available at the end of the article
} 


\section{Background}

Carbon ion therapy is increasingly applied in patients with skull base tumors [1,2]. Although the clinical outcome is quite promising [3-5], a major limitation originates from the limited knowledge of the tolerance doses for late normal tissue reactions in the central nervous system (CNS), which mainly originates from the increased relative biological effectiveness (RBE) of carbon ions as compared to photons. As a result, radiation doses to the tumor and normal tissue are assessed in terms of RBE-weighted rather than absorbed dose [6]. The RBE, however, is a complex quantity and depends critically on the linear energy transfer (LET), on the fractional dose as well as on biological parameters and the considered biological endpoint. In clinical practice, the RBE is predicted quantitatively by biophysical models, such as the local effect model (LEM) [7], and this prediction includes significant uncertainties. Besides clinical investigations, experimental studies in animals have been performed to validate these RBE-models and to depict differences in the development of late CNS reactions between high- and low-LET irradiations.

The RBE of carbon ions in the rat spinal cord was evaluated for the endpoint radiation-induced myelopathy in previous studies [8-11], however, only one data set examined the dependence of the RBE on dose and LET $[8,9]$. In those dose-response studies, irradiations of the spinal cord were performed in the entrance region and in the middle of a $1 \mathrm{~cm}$ spread-out Bragg peak (SOBP) using different fractionation schemes. Comparison of the results with predictions of the clinically used LEM I showed a significant underestimation of the RBE in the SOBP and deviations in the functional dependence on dose in the entrance region. These findings gave rise to further developments and resulted in the more recent version LEM IV [12], which is, however, not yet applied in patients. Since these early studies only covered two extreme LET-conditions, a systematic in vivo evaluation of the accuracy of the two model versions was not possible. Furthermore, although some early histological investigations to decipher radiation-induced myelopathy after carbon ion irradiation exist [13], no systematic studies on the temporal development and no correlation with findings in clinically relevant imaging modalities, such as magnetic resonance imaging (MRI), is presently available.

Within the translationally oriented clinical research group KFO 214 on heavy ion therapy, funded by the German Research Foundation (DFG), the radiation response of the rat spinal cord was extensively investigated. This contribution gives a brief summary of previously published data [14-16] in terms of dose-response curves for the endpoint radiation-induced myelopathy. Additionally, preliminary results of project-related, unpublished studies are presented including an MRIand histology-based study to examine the temporal development of myelopathy. To protect the spinal cord from radiation-induced damage, the impact of an ACE(angiotensin-converting-enzyme) inhibitor was tested.

\section{Methods and materials}

Animals and anesthesia

For the described studies, a total of 597 young adult female Sprague-Dawley (SD) rats (Charles River, Sulzfeld, Germany) were used. Animals were kept under standard conditions at the German Cancer Research Center (DKFZ) animal laboratory facility. For irradiations, rats received gaseous anesthesia with a mixture of $4 \%$ Sevoflurane (Abbott, Wiesbaden, Germany) and 2 1/min oxygen, whereas for the MRI-measurements $2.5 \mathrm{Vol} \%$ Isoflurane (Abbott, Wiesbaden, Germany) in $1.5 \mathrm{l} / \mathrm{min}$ oxygen was used. All experiments were approved by the governmental review committee on animal care (35-9185.81/ G62-08, G117/13, G34/13).

\section{Follow-up and biological endpoint}

After irradiation, animals were monitored once a week for general health condition and weight. Paresis grade II is defined as neurological symptoms by regular dragging of the foot with palmar flexion or dragging of extended foreleg [17]. A preliminary stage is paresis grade I meaning that the rat shows obvious neurological detractions but the animal is still able to use its forelegs.

The biological endpoint was defined as "radiation-induced myelopathy (paresis grade II) within 300 days". Animals showing this endpoint were scored as responder, sacrificed and the spinal cord was processed for histological examinations.

\section{Dose-response studies}

Details of the experimental setup has been described previously [14] and only a brief summary is given here. The rat cervical spinal cord (segments C1-6, field size $10 \times 15$ $\left.\mathrm{mm}^{2}\right)$ was irradiated at 6 different positions $(35,65,80$, 100,120 and $127 \mathrm{~mm}$ ) of a $6 \mathrm{~cm}$ spread-out Bragg peak (SOBP, range 70-130 $\mathrm{mm}$ water-equivalent depth) corresponding to a dose-averaged linear energy transfer (LET) of $16-99 \mathrm{keV} / \mu \mathrm{m}$. The range of the ions was adjusted using appropriate polymethyl-methacrylate (PMMA)-boli placed in front of the animals. Irradiations were performed in groups of 5 animals with increasing dose levels using either 1 or 2 fractions (Fx) to cover $0-100 \%$ response probability. Animal numbers were selected to determine $\mathrm{TD}_{50}$ (dose at $50 \%$ probability of paresis grade II) with a standard error of about 0.5 Gy. Irradiations were performed under identical conditions either at the Helmholtz Center for Heavy Ion Research (GSI, $100 \mathrm{~mm}$ midposition), or (after beam time became available) at the 
Heidelberg Heavy Ion Therapy Center (HIT, all other positions) using the active raster scanning technique [18]. The presented results for 1 and $2 \mathrm{Fx}$ included a total of 464 irradiated rats as well as 10 sham treated controls.

For each fractionation schedule and each position of the spinal cord within the SOBP, a dose-response curve was determined by performing a maximum-likelihood fit of the logistic dose-response model to the actuarial response rates (technical details, see [14, 15]). Based on the $\mathrm{TD}_{50}$-values of photons $[8,9]$ and carbon ions, the RBE was calculated. The experimental RBE was compared to model predictions using the version I and IV of local effect model (LEM) $[7,12]$. RBE-calculations with the LEM were performed with the treatment planning system TRiP (Treatment Planning for Particles [19]) for the experimentally obtained $\mathrm{TD}_{50}$-values.

\section{MRI-based longitudinal study}

To investigate the temporal development of radiationinduced myelopathy, 24 irradiated animals and 7 shamtreated controls were included in an MR-based longitudinal study. Irradiated animals received $6 \mathrm{Fx}$ of either carbon ions (center of $1 \mathrm{~cm}$ SOBP; LET: $91 \mathrm{keV} / \mu \mathrm{m}$ (range, 80$104 \mathrm{keV} / \mu \mathrm{m})$ ) or $6 \mathrm{MV}$ photons using approximately isoeffective total doses of 23 Gy (RBE) or 61 Gy, respectively. Based on our previous study [8], these doses were known to cause radiation-induced myelopathy in all animals.

For imaging, a 1.5 T MRI scanner (Symphony, Siemens, Erlangen) in combination with an in-house made radiofrequency coil was used. To record the initial state, rats were imaged prior to irradiation. After irradiation, rats were monitored monthly and as soon as morphological alterations in the MR-images occurred, the measurement intervals were reduced.

MRI measurements included a T2-weighted sequence (TE $109 \mathrm{~ms}$, TR $4000 \mathrm{~ms}$, FOV $40 \mathrm{~mm}$ ) to detect edema. To prove the onset of a blood-spinal cord barrier (BSCB) disruption a T1-weighted sequence (TE $14 \mathrm{~ms}$, TR $600 \mathrm{~ms}$, FOV $46 \mathrm{~mm}$ ) in combination with contrast agent application $\left(0.2 \mathrm{mmol} / \mathrm{kg}\right.$, Magnevist ${ }^{\circ}$, Bayer, Leverkusen) was used. In addition, a T1-weighted dynamic contrastenhanced (DCE) MR sequence (TE $1.75 \mathrm{~ms}$, TR $373 \mathrm{~ms}$, FOV $150 \mathrm{~mm}$ ) was used to study radiation-induced alterations in blood perfusion. DCE-measurements were evaluated using a pharmacokinetic model $[20,21]$ allowing the determination of the relative plasma volume, $v_{p}$, the relative interstitial volume, $\mathrm{v}_{\mathrm{e}}$ and the volume transfer coefficient $\mathrm{K}_{\text {trans. }}$

\section{Histology}

Animals reaching the endpoint paresis grade II were perfused with a mixture of $4 \%$ paraformaldehyde (PFA) in $0.015 \mathrm{M}$ phosphate buffered saline. The cervical spinal cord C1-6 was dissected out and postfixed overnight.
Cryosections of $8 \mu \mathrm{m}$ thickness were used for a general staining with hemalum/eosin (HE) in combination with Luxol fast blue [22]. Luxol fast blue was used to examine qualitatively the extent of demyelination since the dye attaches to the lipoproteins of the myelin. A reduced signal is assigned with affected areas.

To study the degree of blood vessel perforation, extravasated serum albumin was immunohistochemically visualized. For this, paraffin sections of $8 \mu \mathrm{m}$ thickness were deparaffinized and rehydrated. Endogenous peroxidase activity was blocked with $3 \% \mathrm{H}_{2} \mathrm{O}_{2}$. To unmask antigen sites, an antigen retrieval with sodium citrate buffer ( $\mathrm{pH}$ 6) was performed. Sections were then incubated overnight at $4{ }^{\circ} \mathrm{C}$ with the primary antibody against albumin (Acris, 1:6000 diluted in 3\% bovine serum albumin) followed by incubation with the secondary antibody (Abcam, 1:500, horse raddish peroxidase). $3,3^{\prime}$-diaminobenzidine was used as chromogen. Afterwards the sections were counterstained with Nissl and evaluated by light microscopy.

\section{Radioprotectiva study}

The protective influence of the ACE-inhibitor ramipril ${ }^{\mathrm{m}}$ was investigated in a four-armed dose-response experiment using a total of 88 animals and four sham-treated controls. Animals were irradiated with single doses of carbon ions (center of $6 \mathrm{~cm}$ SOBP; LET: $45 \mathrm{keV} / \mu \mathrm{m}$ ) or 6 MV photons. 4 animals per dose group with increasing dose levels were used to cover $0-100 \%$ response probability. Each modality includes an experimental arm with and without ramipril $^{\mathrm{Tm}}$ administration. The ACE-inhibitor was given immediately after irradiation $(2 \mathrm{mg} / \mathrm{kg} /$ day $)$ via their drinking water (ad libitum) during the full observation time of 300 days.

\section{Results}

The irradiation procedure, MRI follow-up and ACEinhibitor intake was well tolerated by all animals. Rats which had to be excluded during follow-up due to spontaneous development of mammary carcinomas or death due to unknown reasons were considered by an actuarial approach.

\section{Dose-response studies}

Figure 1 summarizes the dose-response curves obtained at the 6 positions within the SOBP after one and two fractions of carbon ions. The corresponding $\mathrm{TD}_{50}$-values decreased significantly with increasing LET and increased with increasing fraction number, i.e. decreasing fractional dose. Figure 2 displays the resulting LETdependence of the RBE after single and split doses. It was found that the RBE increases much stronger after 2 fractions than after single fractions. Comparing the measured RBE-values with predictions of the LEM revealed 


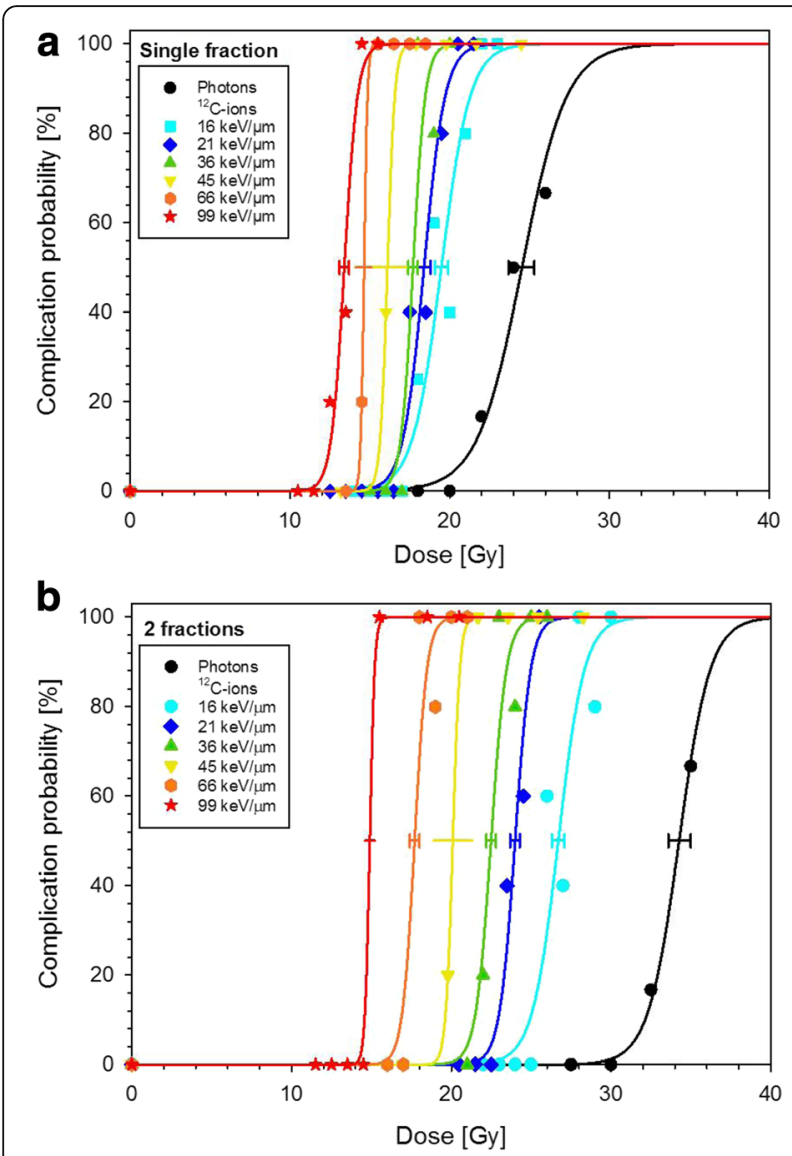

Fig. 1 Dose-response curves for the endpoint paresis grade II after irradiation of the spinal cord with either single (a) or split (b) doses of carbon ions or photons at 6 different positions within a $6 \mathrm{~cm}$ SOBP

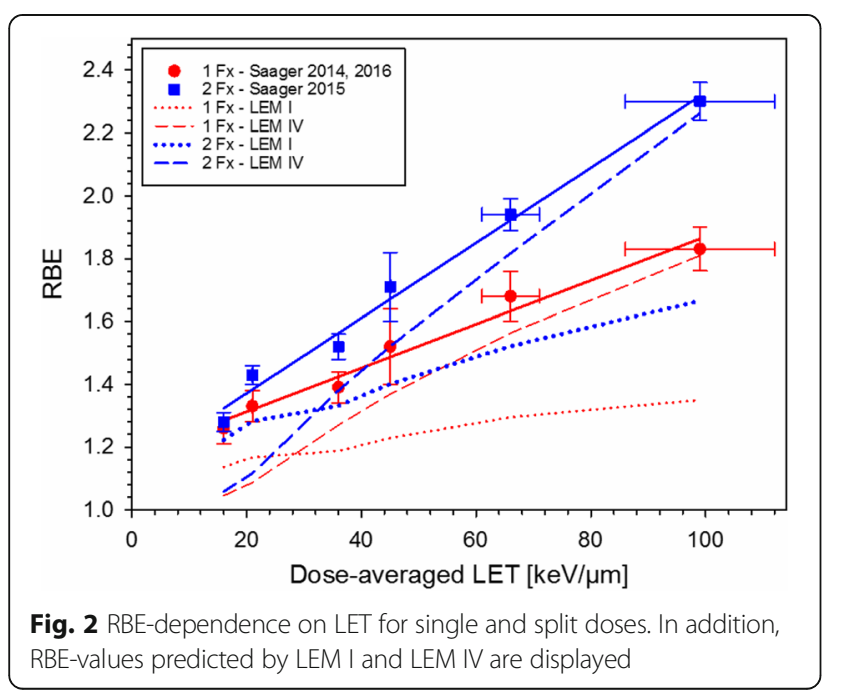

that LEM IV better predicts this stronger increase, and in general provides a much better description in the high-LET region $(30-100 \mathrm{keV} / \mu \mathrm{m})$ of the SOBP while LEM I is more accurate in the low-LET region $(\sim 20 \mathrm{keV} / \mu \mathrm{m})$ of the plateau.

\section{MRI-based longitudinal study}

MRI measurements after carbon ion and photon irradiation revealed the same morphological alterations in the MR images ranging from the development of edema, syrinx (dilatation of the canalis centralis) and contrast agent accumulation up to the final development of the radiation-induced myelopathy (Fig. 3). The latency time until development of paresis grade II, however, was significantly shorter for carbon ions $(136 \pm 10 \mathrm{~d})$ than for photons $(211 \pm 20 \mathrm{~d})$. Evaluation of the DCE-measurements exhibited a continuous increase of the parameters $\mathrm{v}_{\mathrm{e}}$ and $K_{\text {trans }}$ with increasing damage of the BSCB, however, no significant differences between carbon ion and photon irradiation was found, except for the shorter latency time. No significant changes were found for the parameter $\mathrm{v}_{\mathrm{p}}$.

\section{Histology}

After carbon ion as well as after photon irradiation histological examinations of the endpoint paresis grade II revealed a comparable extent of tissue damage (Fig. 4). Compared to the unirradiated control, a structural decline in terms of white matter vacuolization, necrosis, blood vessel dilatation and disruption was found in the posterior and lateral part for both radiation modalities. A clear demyelination represented by the loss of luxol fast blue staining has been seen after photon irradiation (Fig. 4c). The blood vessels in the grey matter were dilated and perforated whereas the overall structure remained visually intact. However, a larger extent of blood vessel perforation was found after carbon ion than after photon irradiation. The albumin extravasation, represented by a brown precipitation, was more intense after carbon ion irradiation, predominately in the dorsal part of the white matter and around the canalis centralis whereas after photon irradiation the albumin extravasation was found to be weaker in these areas (Fig. 4).

\section{Radioprotectiva study}

No protective effect of ramipril ${ }^{\mathrm{Tw}}$ for the development of radiation-induced myelopathy after carbon ion or photon irradiations was observed. However, a modality and dose dependent prolongation of latency time of $23 \pm 8 \mathrm{~d}$ after carbon ion irradiation and $16 \pm 3 \mathrm{~d}$ after photon irradiation was found.

\section{Discussion}

Only very few studies on late effects in normal tissue are presently available [11, 13, 23, 24]. Radiation-induced 


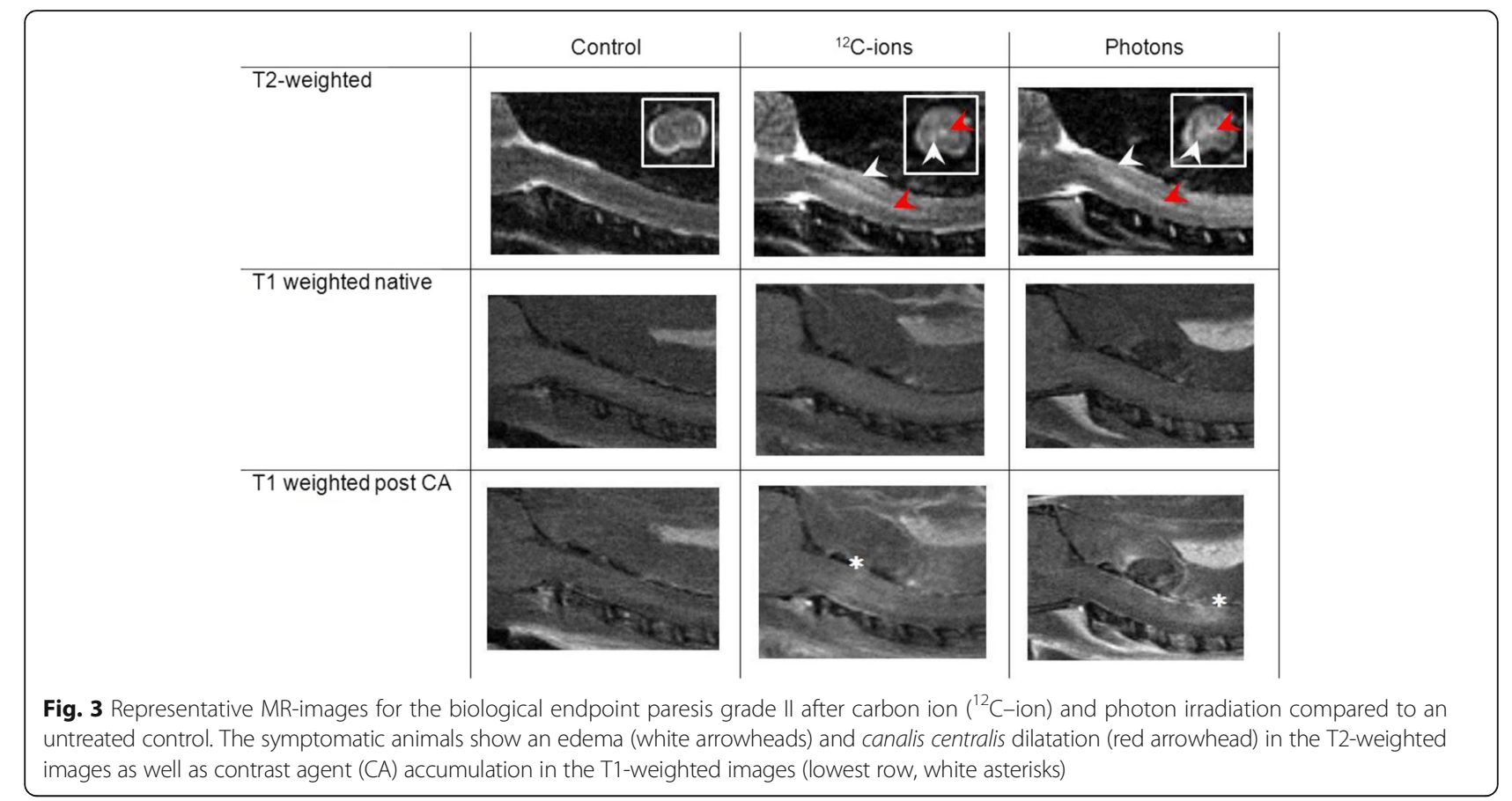

myelopathy is a feared late side effect in the CNS, characterized by a long symptom-free latency period followed by a sudden occurrence of neurological symptoms. To prevent the development of these severe complications, specific tolerance doses have to be respected and due to the uncertainty in the knowledge of the RBE, this is associated with significantly larger uncertainties for carbon ions than for photons.

To investigate the accuracy of RBE-predictions by the LEM, a large-scale dose-response study in the rat spinal cord has been performed. This animal model is well established for the investigation of late effects in the

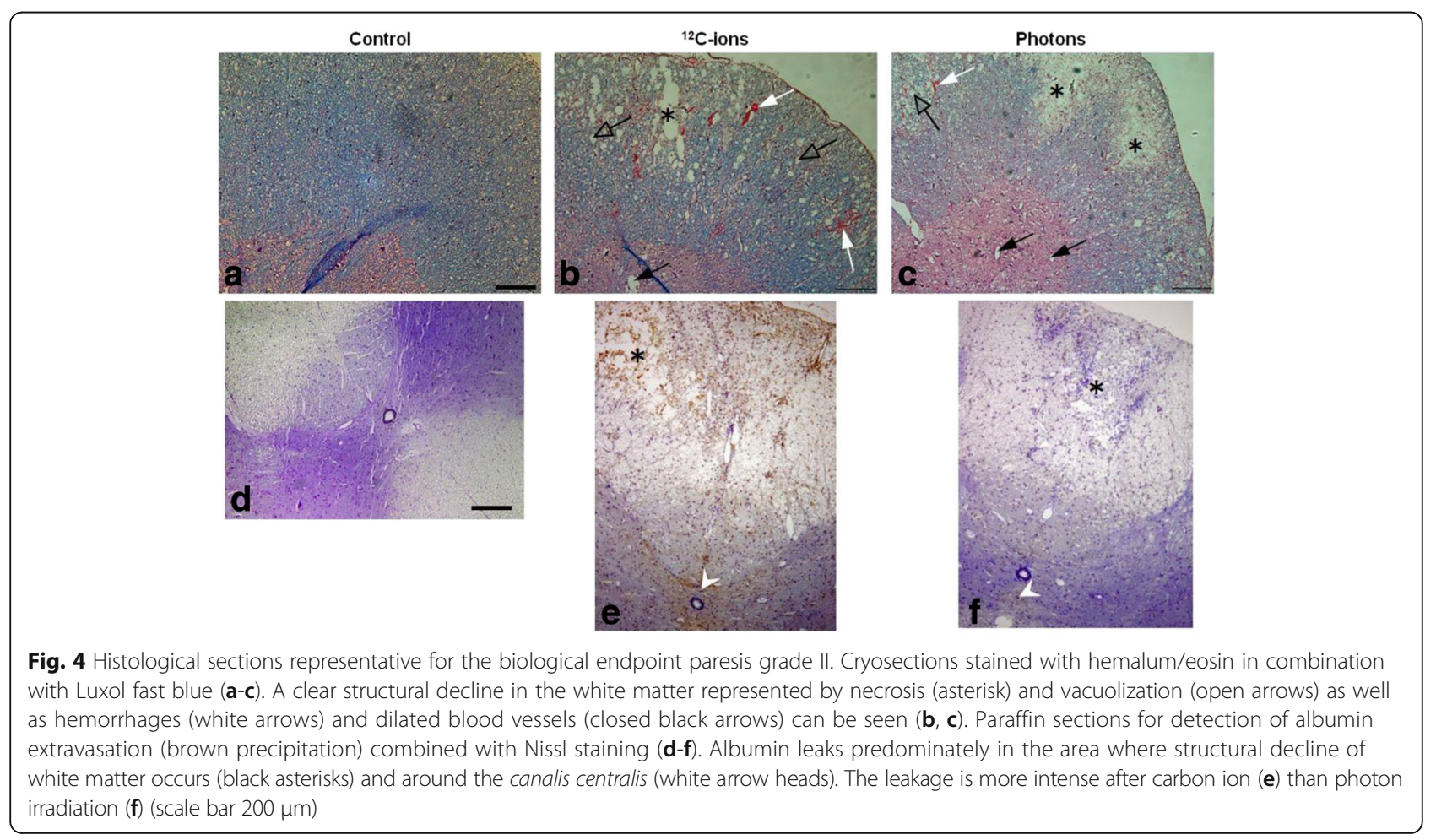


CNS and has been previously used to study the effectiveness of different beam modalities [25-30]. Especially, it has been shown that the response of the spinal cord is independent of the irradiated volume for field lengths above $8 \mathrm{~mm}[31,32]$. The model is also well-suited to study the temporal development of radiation-induced myelopathy in MRI as well as on the histological level. This study currently presents the largest and most systematic data base.

\section{Dose-response studies}

The rat spinal cord was used to characterize the RBEvariation along the central axis of a $6 \mathrm{~cm}$ SOBP for different fractionation schedules. The details of these studies have been published previously [14-16]. Detailed in vivo testing of the RBE-predictions of LEM I and IV as a function of LET and fractional dose revealed that the RBE in the high-LET region is better described by LEM IV while the predictions of LEM I are more accurate in the lowLET region. It has to be noted, however, that this result refers to relatively high fractional doses. An additional dose-response study with $6 \mathrm{Fx}$ is currently under evaluation and will allow to extend the benchmarking of the LEM also towards lower doses per fraction. Together with the presented results, this study will allow to estimate the $\alpha / \beta$-value, which represents the extent of tissue regeneration in fractionated treatments. Preliminary results based on the single and split dose studies suggest an increase of $\alpha / \beta$ with increasing LET, indicating a decreasing impact of fractionation for increasing LET. For a more reliable estimation, however, the 6 Fx study has to be included. It has to be emphasized that the benchmarking of RBE-models is not restricted to the LEM. Currently, tests are extended to the Microdosimetric Kinetic Model (MKM) which is used for carbon ion therapy at National Institute of Radiological Science (NIRS, [33, 34]).

\section{MRI-based longitudinal study}

The MRI-based longitudinal study enables a non-invasive investigation of occurring radiation-induced effects during the symptom free latency time. We found a fixed sequence of alterations in the images. Comparing the carbon ion and photon irradiations at isoeffective doses with respect to the endpoint paresis grade II, the same morphological changes were found and the only difference was a shorter latency time after carbon ion irradiation. Main findings in MRI were presence of edema, syrinx, uptake of contrast agent due to the break-down of the BSCB and finally followed by paresis grade I and II. Once the edema occurred in an animal, it developed the deterministic sequence. These findings were also confirmed quantitatively by evaluation of the DCE-measurements, which showed that the increase of the extracellular volume, $\mathrm{v}_{\mathrm{e}}$, and the contrast agent exchange rate, $K_{\text {trans }}$, increased similarly for carbon ions and photons.

It appears likely that the shorter latency time after carbon ion irradiations originates from differential actions on the histological or molecular level and apparently, MRI at 1.5 $\mathrm{T}$ is not sensitive enough for the detection of such alterations. With respect to sensitivity, the small diameter of the rat spinal cord and the consequently occurring partial volume effects may also play a role. Using an MRI with higher field strength would in principle be an option to increase the sensitivity, yet, in the present study, this was logistically not possible due to the excessive number of measurements, which had to be performed on a short term notice during the period where neurological symptoms appear within a rapid time sequence.

Despite of these limitations, this study provides the first extensive temporal characterization of the development of radiation-induced myelopathy after irradiation with carbon ions and photons in MRI and in an ongoing MRI-based histological study, tissue samples at different time points after irradiation as well as at the occurrence of the different endpoints in MRI are acquired. By investigating these samples on the histological and molecular level, more detailed information on the underlying mechanistic processes is expected.

\section{Molecular mechanisms and inhibition}

Currently, it is not clear in detail whether the target structures of irradiation in the spinal cord are the neurons or the blood vessels. Therefore, many attempts have been made to evaluate the effects of ionizing radiation to the neuronal [22, 35-37] and the vascular proportion $[11,24,38-41]$ supporting nowadays the view that endothelial cells are the main target structure [42-44].

At the endpoint paresis grade II, histological examinations revealed a comparable breakdown of the tissue structure for both radiation modalities; however, the increase of blood vessel permeability was much higher after carbon ion irradiation. This finding is in contrast to results of the DCE evaluation, where no difference was seen at the same endpoint.

It has to be noted, however, that increased permeability of the BSCB was detected with albumin, which presents a much larger molecule than MRI contrast agent Gd-DTPA ( 66 vs. $0.5 \mathrm{kDa}$ ). The discrepancy between the results of MRI and histological analysis could therefore be explained by a different extent of perforation for the two irradiation modalities. While the higher ionization density of carbon ions introduces more complex, nonreparable DNA-damage, which leads to intense blood vessel perforation and thus to an increased permeability for Gd-DTPA as well as for albumin, photons exhibit a low ionization density which induces better reparable 
DNA-damage and leads only to small vessel perforations and thus to an increased permeability for Gd-DTPA but much less for albumin. To clarify this, additional histological investigations with smaller molecular markers are required.

Besides vascular changes, also a profound damage of the neuronal structures was observed. Luxol fast blue staining shows a clear decrease of the myelin basic protein at the biological endpoint paresis grade II. To assess the relative importance of vascular and neuronal damage, a detailed investigation of the temporal development of both structures on the histological and molecular level will be performed within the ongoing MRI-based histological study.

Detailed knowledge of the mechanistic processes may enable targeted pharmacological interventions with the aim of protecting the normal central nervous system tissue after irradiation. First attempts along this direction have already been described in the literature [45-48] using ACE-inhibitors. Within a pilot trial, we used the ACE-inhibitor ramipril ${ }^{\text {tw }}$ to test the impact on radiationinduced myelopathy after carbon ion and photon irradiation. The rationale for using this drug are manifold: ramipril $^{\text {mis }}$ has been shown to exhibit mitigative properties on optic neuropathy [47, 49]. In addition, with regard to the central nervous system, the drug is able to cross the blood-spinal cord barrier [50], does not reveal protective effects on tumors [51] and is already used to treat hypertension in patients. Our results showed that myelopathy could not be prevented, however a prolongation of latency time was achieved, which indicates that ramipril ${ }^{\text {Th }}$ has a mitigative effect in the rat spinal cord. Identification of the underlying pathological pathways leading to radiation-induced side effects would facilitate the application of appropriate protective drugs and, if successfully realized, could allow elevating the tumor dose without harming the surrounding normal tissue.

\section{Conclusion}

Within this study, a large data base on RBE for late effects in CNS tissue of the rat after carbon ion irradiation was established and used for benchmarking the functional dependencies of the RBE on LET and dose as predicted by LEM I and LEM IV. According to this comparison, LEM IV better describes the measured data in the high-LET region while LEM I predictions are more accurate in the low-LET region. Ongoing studies will extend this database further. Using MRI, an extensive characterization of the temporal development of radiation-induced alterations in the rat spinal cord was obtained. The main result was a shorter latency time for carbon ions than for photons. This finding is expected to originate from complex pathological pathways on the molecular level, which needs further investigations. This hypothesis is supported by histological investigations, where an increased vessel perforation, associated with a differential pattern of permeability, was found after carbon ion as compared to photon irradiations. For the ACE-inhibitor ramipril ${ }^{\text {rim }}$, a mitigative rather than protective effect was found, however, the design of targeted protective drugs requires more detailed knowledge on the molecular pathways during the pathogenesis of radiation-induced myelopathy.

\section{Abbreviations \\ ${ }^{12}$ C-ion: Carbon ion; ACE: Angiotensin-converting-enzyme; BSCB: Blood- spinal cord barrier; CNS: Central nervous system; DCE: Dynamic contrast enhanced; FOV: Field of view; Gd-DTPA: Gadolinium diethylenetriaminepentacetate; LEM: Local effect model; LET: Linear energy transfer; MKM: Microdosimetric kinetic model; MRI: Magnetic resonance imaging; NIRS: National Institute of Radiological Science; RBE: Relative biological effectiveness; SD: Sprague Dawley; SOBP: Spread-out Bragg Peak; TD: Tolerance dose; TE: Echo time; TR: Repetition time; TRiP: Treatment planning for particles}

\section{Acknowledgements}

The authors would like to thank the DKFZ Core Facility 'Small Animal Imaging Center', especially Dr. M. Jugold and I. Babushkina. This work was supported by the German Research Foundation (DFG, KFO 214).

\section{Funding}

The study was funded by the German Research Foundation (DFG; KFO 214).

\section{Availability of data and materials}

The datasets analysed during the current study are available from the corresponding author on reasonable request.

\section{Authors' contributions}

MS organized and carried out the experiments (dose-response study, MRI study, radioprotectiva study), established histological protocols and performed histological evaluation of the rat spinal cord and had the responsibility for the animals. She wrote animal proposals and the manuscript. PP initiated the project and was involved in the study design, data interpretation and writing. TW evaluated the MR-images. LH evaluated the DCE-MRI data. SB supported the high-LET irradiations at the HIT facility. RG performed the LEM calculations. MS performed the LEM calculations. JD initiated the project and was involved in the translational assessment of the data. CK initiated the project and was involved in the experimental design, dosimetry, treatment planning and statistical evaluation. All authors read and approved the final manuscript.

\section{Ethics approval}

All experiments were approved by the governmental review committee on animal care (35-9185.81/G62-08, G117/13, G34/13).

\section{Consent for publication}

Not applicable.

\section{Competing interests}

The authors declare that they have no competing interests.

\section{Publisher's Note}

Springer Nature remains neutral with regard to jurisdictional claims in published maps and institutional affiliations.

\section{Author details}

${ }^{1}$ Department of Radiation Oncology, University Hospital of Heidelberg, Heidelberg, Germany. ${ }^{2}$ Department of Medical Physics in Radiation Oncology (E040), German Cancer Research Center (DKFZ), Im Neuenheimer Feld 280, 69120 Heidelberg, Germany. ${ }^{3}$ National Center for Radiation Research in Oncology (NCRO), Heidelberg Institute for Radiation Oncology (HIRO), Heidelberg, Germany. ${ }^{4}$ Clinical Cooperation Unit Molecular Radiooncology, 
German Cancer Research Center (DKFZ), Heidelberg, Germany. ${ }^{5}$ Heidelberg Ion Beam Therapy Center (HIT), Heidelberg, Germany. ${ }^{6}$ Department of Biophysics, Helmholtz Center for Heavy Ion Research (GSI), Darmstadt, Germany.

Received: 4 August 2017 Accepted: 29 December 2017

Published online: 11 January 2018

\section{References}

1. Uhl M, Herfarth K, Debus J. Comparing the use of protons and carbon ions for treatment. Cancer J. 2014;20(6):433-9.

2. Mizoe JE. Review of carbon ion radiotherapy for skull base tumors (especially chordomas). Rep Pract Oncol Radiother. 2016;21(4):356-60.

3. Jensen AD, Nikoghosyan AV, Poulakis M, et al. Combined intensitymodulated radiotherapy plus raster-scanned carbon ion boost for advanced adenoid cystic carcinoma of the head and neck results in superior locoregional control and overall survival. Cancer. 2015;121(17):3001-9.

4. Uhl M, Mattke M, Welzel T, et al. High control rate in patients with chondrosarcoma of the skull base after carbon ion therapy: first report of long-term results. Cancer. 2014;120(10):1579-85.

5. Uhl M, Mattke M, Welzel T, et al. Highly effective treatment of skull base chordoma with carbon ion irradiation using a raster scan technique in 155 patients: first long-term results. Cancer. 2014;120(21):3410-7.

6. Kraft G. Tumor therapy with heavy charged particles. Prog Part Nucl Phys. 2000;45:5473-544.

7. Scholz M, Kellerer AM, Kraft-Weyrather W, Kraft G. Computation of cell survival in heavy ion beams for therapy. The model and its approximation. Radiat Environ Biophys. 1997;36(1):59-66.

8. Debus J, Scholz M, Haberer T, et al. Radiation tolerance of the rat spinal cord after single and split doses of photons and carbon ions. Radiat Res. 2003;160(5):536-42.

9. Karger CP, Peschke P, Sanchez-Brandelik R, Scholz M, Debus J. Radiation tolerance of the rat spinal cord after 6 and 18 fractions of photons and carbon ions: experimental results and clinical implications. Int J Radiat Oncol Biol Phys. 2006;66(5):1488-97.

10. Leith JT, McDonald M, Powers-Risius P, Bliven SF, Howard J. Response of rat spinal cord to single and fractionated doses of accelerated heavy ions. Radiat Res. 1982;89(1):176-93.

11. Okada S, Okeda R, Matsushita S, Kawano A. Histopathological and morphometric study of the late effects of heavy-ion irradiation on the spinal cord of the rat. Radiat Res. 1998;150(3):304-15.

12. Elsässer $T$, Weyrather WK, Friedrich $T$, et al. Quantification of the relative biological effectiveness for ion beam radiotherapy: direct experimental comparison of proton and carbon ion beams and a novel approach for treatment planning. Int J Radiat Oncol Biol Phys. 2010;78(4):1177-83.

13. Okada S, Okeda R. Pathology of radiation myelopathy. Neuropathology. 2001:21(4):247-65.

14. Saager $M$, Glowa $C$, Peschke $P$, et al. Carbon ion irradiation of the rat spinal cord: dependence of the relative biological effectiveness on linear energy transfer. Int J Radiat Oncol Biol Phys. 2014;90(1):63-70.

15. Saager M, Glowa C, Peschke $P$, et al. Split dose carbon ion irradiation of the rat spinal cord: dependence of the relative biological effectiveness on dose and linear energy transfer. Radiother Oncol. 2015;117(2):358-63.

16. Saager M, Glowa C, Peschke $P$, et al. The relative biological effectiveness of carbon ion irradiations of the rat spinal cord increases linearly with LET up to $99 \mathrm{keV} /$ mum. Acta Oncol. 2016;55(12):1512-5.

17. Ruifrok AC, Stephens LC, van der Kogel AJ. Radiation response of the rat cervical spinal cord after irradiation at different ages: tolerance, latency and pathology. Int J Radiat Oncol Biol Phys. 1994;29(1):73-9.

18. Haberer T, Becher W, Schardt D, Kraft G. Magnetic scanning system for heavy ion therapy. Nuclear Instruments and Methods in Physics Research Section A: Accelerators, Spectrometers, Detectors and Associated Equipment. 1993;330(1):296-305.

19. Kramer M, Jakel O, Haberer T, et al. Treatment planning for heavy-ion radiotherapy: physical beam model and dose optimization. Phys Med Biol. 2000;45(11):3299-317.

20. Tofts PS, Brix G, Buckley DL, et al. Estimating kinetic parameters from dynamic contrast-enhanced T(1)-weighted MRI of a diffusable tracer: standardized quantities and symbols. J Magn Reson Imaging. 1999;10(3): 223-32.
21. Tofts PS. Modeling tracer kinetics in dynamic Gd-DTPA MR imaging. J Magn Reson Imaging. 1997;7(1):91-101.

22. Atkinson S, Li YQ, Wong CS. Changes in oligodendrocytes and myelin gene expression after radiation in the rodent spinal cord. Int J Radiat Oncol Biol Phys. 2003;57(4):1093-100.

23. Sorensen BS, Horsman MR, Alsner J, et al. Relative biological effectiveness of carbon ions for tumor control, acute skin damage and late radiation-induced fibrosis in a mouse model. Acta Oncol. 2015: 54(9):1623-30.

24. Okada S, Okeda R, Matsushita S, Kawano A. Immunohistochemical changes of the blood-brain barrier in rat spinal cord after heavy-ion irradiation. Neuropathology. 1998;18(2):188-98.

25. Bijl HP, van Luijk P, Coppes RP, et al. Regional differences in radiosensitivity across the rat cervical spinal cord. Int J Radiat Oncol Biol Phys. 2005;61(2):543-51.

26. Bijl HP, van Luijk P, Coppes RP, et al. Influence of adjacent low-dose fields on tolerance to high doses of protons in rat cervical spinal cord. Int J Radiat Oncol Biol Phys. 2006;64(4):1204-10.

27. Leith JT, Lewinsky BS, Woodruff KH, Schilling WA, Lyman JT. Tolerance of the spinal cord of rats to irradiation with cyclotron-accelerated heliumions. Cancer. 1975:35(6):1692-700.

28. Ruifrok AC, Kleiboer BJ, van der Kogel AJ. Radiation tolerance and fractionation sensitivity of the developing rat cervical spinal cord. Int J Radiat Oncol Biol Phys. 1992;24(3):505-10.

29. van der Kogel AJ, Barendsen GW. Late effects of spinal cord irradiation with $300 \mathrm{kV}$ X rays and $15 \mathrm{MeV}$ neutrons. Br J Radiol. 1974;47(559):393-8.

30. van der Kogel AJ, Sissingh HA, Zoetelief J. Effect of $X$ rays and neutrons on repair and regeneration in the rat spinal cord. Int J Radiat Oncol Biol Phys. 1982;8(12):2095-7.

31. Bijl HP, van Luijk P, Coppes RP, et al. Dose-volume effects in the rat cervical spinal cord after proton irradiation. Int J Radiat Oncol Biol Phys. 2002;52(1):205-11.

32. Hopewell JW, Morris AD, Dixon-Brown A. The influence of field size on the late tolerance of the rat spinal cord to single doses of $\mathrm{X}$ rays. $\mathrm{Br} J$ Radiol. 1987;60(719):1099-108.

33. Inaniwa T, Furukawa T, Kase Y, et al. Treatment planning for a scanned carbon beam with a modified microdosimetric kinetic model. Phys Med Biol. 2010;55(22):6721-37.

34. Inaniwa T, Kanematsu N. A trichrome beam model for biological dose calculation in scanned carbon-ion radiotherapy treatment planning. Phys Med Biol. 2015;60(1):437-51.

35. Wei L, Zhou Y, Liu C-J, Zheng K, You H. Demyelination occurred as the secondary damage following diffuse axonal loss in a rat model of radiation myelopathy. Neurochem Res. 2017:42(4):953-62.

36. Atkinson SL, Li YQ, Wong CS. Apoptosis and proliferation of oligodendrocyte progenitor cells in the irradiated rodent spinal cord. Int J Radiat Oncol Biol Phys. 2005;62(2):535-44.

37. Li YQ, Jay V, Wong CS. Oligodendrocytes in the adult rat spinal cord undergo radiation-induced apoptosis. Cancer Res. 1996;56(23):5417-22.

38. Li YQ, Chen P, Haimovitz-Friedman A, Reilly RM, Wong CS. Endothelial apoptosis initiates acute blood-brain barrier disruption after ionizing radiation. Cancer Res. 2003;63(18):5950-6.

39. Li YQ, Chen P, Jain V, Reilly RM, Wong CS. Early radiation-induced endothelial cell loss and blood-spinal cord barrier breakdown in the rat spinal cord. Radiat Res. 2004;161(2):143-52.

40. Lyubimova N, Hopewell JW. Experimental evidence to support the hypothesis that damage to vascular endothelium plays the primary role in the development of late radiation-induced CNS injury. Br J Radiol. 2004; 77(918):488-92.

41. Zhang J, Wei L, Sun WL, et al. Radiation-induced endothelial cell loss and reduction of the relative magnitude of the blood flow in the rat spinal cord. Brain Res. 2014;1583:193-200.

42. Coderre JA, Morris GM, Micca PL, et al. Late effects of radiation on the central nervous system: role of vascular endothelial damage and glial stem cell survival. Radiat Res. 2006;166(3):495-503.

43. Morris GM, Coderre JA, Whitehouse EM, Micca P, Hopewell JW. Boron neutron capture therapy: a guide to the understanding of the pathogenesis of late radiation damage to the rat spinal cord. Int J Radiat Oncol Biol Phys. 1994;28(5):1107-12.

44. Morris GM, Coderre JA, Bywaters A, Whitehouse E, Hopewell JW. Boron neutron capture irradiation of the rat spinal cord: histopathological 
evidence of a vascular-mediated pathogenesis. Radiat Res. 1996;146(3): 313-20.

45. Jenrow KA, Brown SL, Liu J, et al. Ramipril mitigates radiation-induced impairment of neurogenesis in the rat dentate gyrus. Radiat Oncol. 2010;5:6.

46. Jenrow KA, Liu J, Brown SL, et al. Combined atorvastatin and ramipril mitigate radiation-induced impairment of dentate gyrus neurogenesis. J Neuro-Oncol. 2011;101(3):449-56.

47. Kim JH, Brown SL, Kolozsvary A, et al. Modification of radiation injury by ramipril, inhibitor of angiotensin-converting enzyme, on optic neuropathy in the rat. Radiat Res. 2004;161(2):137-42.

48. Ryu S, Kolozsvary A, Jenrow KA, Brown SL, Kim JH. Mitigation of radiationinduced optic neuropathy in rats by ACE inhibitor ramipril: importance of ramipril dose and treatment time. J Neuro-Oncol. 2007:82(2):119-24.

49. Kim JS, Yun I, Choi YB, Lee KS, Kim YI. Ramipril protects from free radical induced white matter damage in chronic hypoperfusion in the rat. J Clin Neurosci. 2008;15(2):174-8.

50. Nordstrom M, Abrahamsson T, Ervik M, Forshult E, Regardh CG. Central nervous and systemic kinetics of ramipril and ramiprilat in the conscious dog. J Pharmacol Exp Ther. 1993;266(1):147-52

51. Juillerat-Jeanneret L, Celerier J, Chapuis Bernasconi C, et al. Renin and angiotensinogen expression and functions in growth and apoptosis of human glioblastoma. Br J Cancer. 2004:90(5):1059-68.

Submit your next manuscript to BioMed Central and we will help you at every step:

- We accept pre-submission inquiries

- Our selector tool helps you to find the most relevant journal

- We provide round the clock customer support

- Convenient online submission

- Thorough peer review

- Inclusion in PubMed and all major indexing services

- Maximum visibility for your research

Submit your manuscript at www.biomedcentral.com/submit
Biomed Central 